\title{
THE POSSIBILITY OF BETA DECAY IN THE ATMOSPHERES OF NUCLEI OF PLANETARY NEBULAE
}

\author{
G. A. Gurzadian \\ (Branch of Byurakan Astrophysical Observatory, U.S.S.R.)
}

\begin{abstract}
At present there is some basis for questioning the usual presentation of the nuclei of planetary nebulae as being ordinary stars. If the quantity of mass released by a star during its formation or at some stage of its evolution serves as a criterion for its not being stationary, then the nuclei of planetary nebulae are hardly the most nonstationary objects. A strong deviation from Planck's radiation law may also serve as a criterion for non-stationarity when the deviation occurs in the far short-wave region of the spectrum in nuclei and similar objects (e.g. stars of Wolf-Rayet which are not nuclei of planetary nebulae). Finally, the existence of synchrotron radio radiation in some planetary nebulae must be explained by the exceptional activity of their nuclei. It would seem that the nuclei of these nebulae are suppliers if not of typical relativistic electrons then at least of electrons with high energies.
\end{abstract}

In the present report an attempt will be made to show that between these phenomena, there probably exists some common trait and these phenomena may be understood if the existence or appearance of so-called fast electrons is assumed (i.e. electrons with energies of the order $10^{6}-10^{7} \mathrm{eV}$ ) in the outer regions of the atmosphere of the nucleus.

Primary attention in this article will, however, be devoted to the deviation from Planck's law of the radiation in the short-wavelength region of the spectrum of the nucleus.

One must consider as one of the important results of recent color observations of the nuclei of planetary nebulae the establishment of positive color indexes of a number of nuclei. Thus, according to data by Abell (1966), the color index of the nucleus of the nebula A 32 is +0 . 62 , and that of A 37 is +0 . 56 . In these cases it is not necessary to consider the influence of interstellar reddening because the Galactic declination of the first nebula is $+33^{\circ} .8$, and of the second $+42^{\circ} .2$. Among the remaining objects of Abell's list, even after introducing corrections for the effect of interstellar reddening, the color index of at least 10 nuclei appeared within the range of +0.3 to +0 . $^{\mathrm{m}} 8$. The existence of red nuclei was also determined by Kazarian (1967) in a number of nebulae (e.g. for the nucleus of nebula V-V 421 the color index, corrected for interstellar extinction, is equal to +0.32 ). Stars with such color indexes cannot cause nebular luminescence. If we do not take into account the rather trivial assumption that the

Osterbrock and O'Dell (eds.), Planetary Nebulac, 332 338. ' I.A.U. 
luminescence of the indicated nebulae may be caused by invisible hot components in the nuclei, then one must conclude that there is a great excess of luminescence in the short-wave region of the spectrum of these cold nuclei.

Let us consider another example. Among the nuclei of planetary nebulae we meet very many stars of the Wolf-Rayet type which are apparently different from the field Wolf-Rayet stars only in their brightness, being identical with them in other respects. However, it is known that the excitation temperatures found with the help of emission lines of various ions greatly vary from one another even within one particular star. In this case one observes a rather well-pronounced effect in that the excitation temperature rises with increased ionization potential of the atom. Figure 1 illustrates the

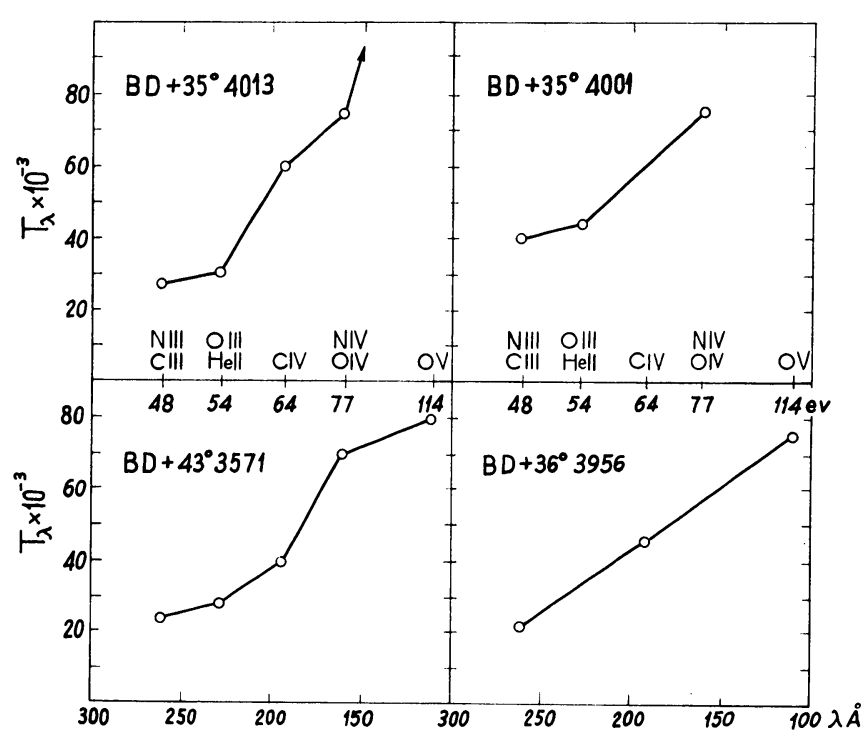

Fig. 1. Color temperatures of central stars determined for several ions, plotted against the wavelength at the ionization boundary. The ionization potential is also shown.

dependence of excitation temperature $T_{*}$ on ionization potential (in this case as a function of the boundary wavelength $\lambda$ of the ionization) for a few Wolf-Rayet stars. These diagrams are derived using Aller's (1943) data. Such a result is qualitatively obtained also when the temperature is determined by the method of Zanstra.

A number of attempts have been made to show the causes or factors which, if not considered when determining the temperature of Wolf-Rayet stars, might lead to such a result (e.g. increase of temperature with the increase of ionization potential). A detailed survey of the latter point has been made by Solobev. However, it is unlikely that future improvements of methods of temperature determination of Wolf- 
Rayet stars, taking into account all possible factors, could introduce any basic change in the general character of the above regularity.

Therefore, by admitting that the increase of temperature in the short-wave region of the spectrum really takes place, one must conclude that a deviation from the perfect radiator exists in these stars and that the effect is greater at increasingly higher frequencies.

The inverse Compton-effect might be a possibility as a mechanism leading to such deviations, i.e. the scattering of light quanta on fast electrons with energies $E$, of the order of $10^{6}-10^{7} \mathrm{eV}$. A formal examination of this process as applied to stellar atmospheres has been made by Gurzadian (1965).

Let us assume that the release or outflow of gaseous matter from the interior of Wolf-Rayet stars is accompanied by the appearance of fast electrons in its atmosphere. The scattering of photons by fast electrons takes place with an increase in frequency according to the correlation $v \approx v^{\prime}\left(E / m c^{2}\right)^{2}$, where $v$ and $v^{\prime}$ are the frequencies of the photon before and after collision with an electron.

Let us designate through $\tau$ the effective optical depth in fast electrons of the layer: $\tau=\sigma_{\mathrm{e}} N$, where $\sigma_{\mathrm{e}}=0.665 \times 10^{-24} \mathrm{~cm}^{2}, N$ is the total number of fast electrons in the column with the base $1 \mathrm{~cm}^{2}$. The layer of fast electrons may be immediately on the photosphere or far from it. Let us assume that the intensity of the thermal radiation released from the photosphere at the frequency $v$ follows Planck's formula and is equal to $B_{v}\left(T_{0}\right)$, where $T_{0}$ is the effective temperature of the photosphere: $B_{v}\left(T_{0}\right)$ is simultaneously the intensity at the base of the layer of fast electrons, where $\tau=0$.

Further assuming that the indicated layer consists of mono-energetic electrons, i.e. the energy of all electrons is equal to $E$, we shall have for the intensity $I_{v}(\mu, \tau)$ emitted from such a layer in some frequency $v$ (see details in Gurzadian, 1965):

where

$$
I_{v}(\mu, \tau)=B_{v}\left(T_{0}\right) C_{v}(\mu, \tau)
$$

$C_{v}(\mu, \tau)=\left(1+\begin{array}{ccc}1 & 1 & \exp \left(x_{0}\right)-1 \\ 4 \pi \mu^{4} \exp \left(x_{0} / \mu^{2}\right)-1 & \tau\end{array}\right) e^{-\tau}$,

and where

$$
\mu=E / m c^{2} ; \quad x_{0}=h v / \kappa T_{0} .
$$

From (1) and (2) follows, that as a result of the scattering of fast electrons on light quanta issuing from the photosphere (or from the atmosphere of the star) an increase in the intensity of the short-wave region of the spectrum takes place at the expense of its weakening in the long-wave region. The excess of the energy in the shortwave region of the spectrum, naturally, leads to a rise of the effective temperature in that region.

In order to obtain a quantitative estimate of the growth of the temperature in the various regions of the spectrum, let us substitute the function of $I_{v}(\mu, \tau)$ in (1) by 
using Planck's function at some effective temperature $T_{v}$ ai a frequency $v$. Putting also the expression $B_{v}\left(T_{0}\right)$ in (1) we obtain:

$$
e^{x}=1+\left[1+\frac{1}{4 \pi \mu^{4}} \frac{\exp \left(x_{0}\right)-1}{\exp \left(x_{0} / \mu^{2}\right)-1} \tau\right]^{-1}\left[\exp \left(x_{0}\right)-1\right] e^{\tau},
$$

where $x=h v / \kappa T_{v}$, and $T_{v}$ is the new temperature. In the regions of short waves (essentially those at $\lambda<1000 \AA$ ) we shall have from (3) to a sufficient degree of approximation:

$$
T_{\lambda}=T_{0} \frac{\mu^{2}}{1+(\lambda \kappa / h c)\left(\ln 4 \pi \mu^{4}+\ln e^{\tau} \tau^{-1}\right) \mu^{2} T_{0}} .
$$

Thus, if $T_{0}, \tau$ and $\mu$ are known, then with the help of (3) or (4) we can determine the effective temperature $T_{\lambda}$ as it changes with wavelength.

In a circuitous manner the dependence of $T_{\lambda}$ on $\lambda$ was determined at three values of $T_{0}\left(10000^{\circ}, 20000^{\circ}\right.$, and $\left.30000^{\circ}\right)$ in the limits of $\tau$ from 0.1 to 0.00001 and at $\mu^{2}=10$, which corresponds to the energy of fast electrons of the order of $1.5 \times 10^{6} \mathrm{eV}$. The results of the calculations are shown in Figure 2. The ionization boundaries of various ions are also indicated there.

As shown in the figures, the effective temperature in the short-wavelength regions shorter than $300 \AA$ - increases several times over the original temperature. In addition, the effective temperature increases monotonically with increasing frequency, i.e. with the growth of the ionization potential. It is characteristic that the effective temperature in the short-wave region can, according to observations (see Figure 1), increase the temperature of a star by a factor of 2 or more in the long-wavelength region even at very small values of the optical thickness of the layer of fast electrons, i.e. when $\tau \sim 10^{-4}-10^{-5}$. This ultraviolet excess is not of thermal origin. Therefore, it is seen that excitation, typical of gaseous nebulae and expanded envelopes of stars, is expected from a comparatively cold star if it is surrounded by a cloud of fast electrons.

It seems to us that the appearance of fast electrons on the outer regions of the atmosphere of a star is a general phenomenon appearing in all or almost all stars. For example, in the case of dwarfs of late spectral types, the presence of fast electrons may lead to a sharp increase of the brightness of a star in the visible ultraviolet region of the spectrum, to excitation of emission lines, etc. (these questions are examined in detail by Gurzadian, 1966). In the case of the Sun the hypothesis of fast electrons explains the observed spectra of X-rays of non-thermal origin (X-ray flares), etc. In the case of those hot stars which are the nuclei of planetary nebulae, the usual Wolf-Rayet stars and those of type $\mathrm{O}$, the fast electrons lead to a sharp increase of the far ultraviolet end of their spectrum.

The truly remarkable thing is that in all of the above cases the best accordance, both qualitatively and quantitatively, of the theory with observations is obtained with $\mu^{2} \simeq 10$, i.e. in the presence of fast electron energies of the order of $10^{6}-10^{7} \mathrm{eV}$. This 

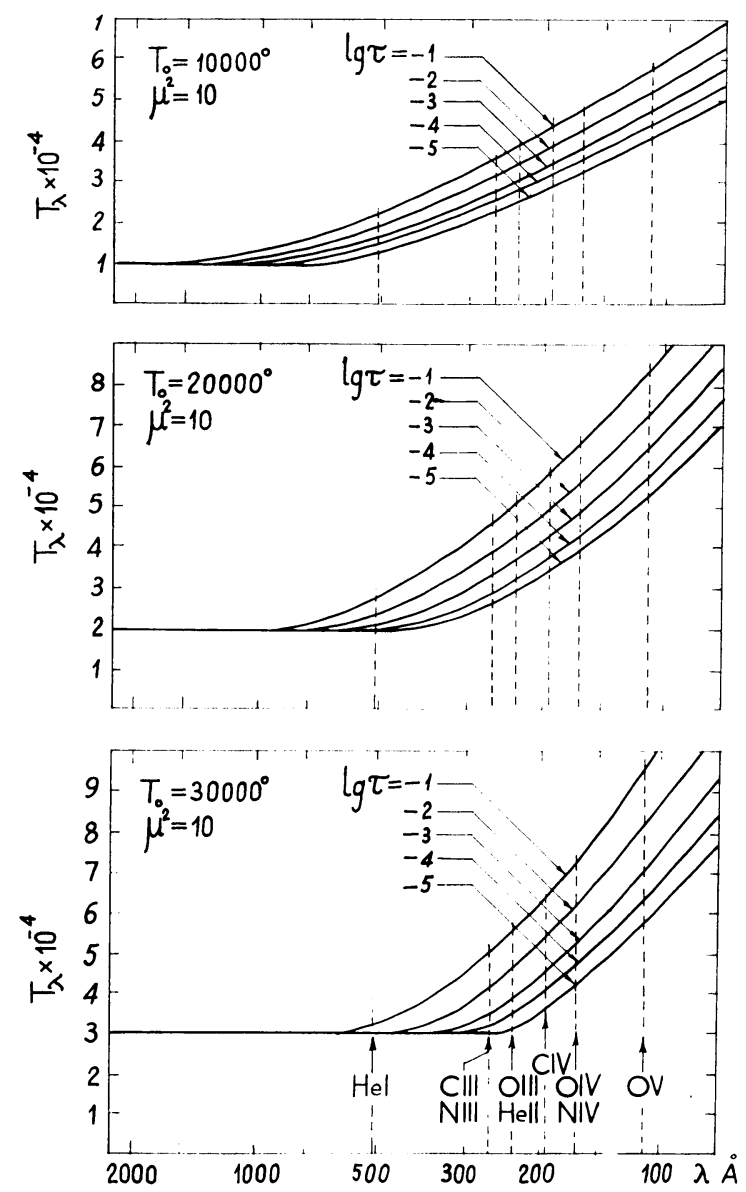

FIG. 2. Theoretical color temperatures for atmospheres optically thick to fast electron scattering.

energy is the order of the electron energy of $\beta$-decay of some unstable nuclei.

Ambartsumian (1954) showed that continual emission is connected with the wide range of phenomena observed in various non-stable objects, and pointed out the non-thermal nature of this emission. Refraining from suggesting a concrete physical mechanism, which might have led to the generation of this emission, Ambartsumian did, however, indicate the possibility of transporting energy in definite amounts from the internal parts of the stars to the outside layers by means of some mechanism unknown to us.

The above considerations allow us to draw the following conclusion: the carriers of stellar interior energy may be fast electrons which appear on the outer regions of 
the atmospheres of stars as a result of the $\beta$-decay of non-stable nuclei or nucleons. The latter, in turn, are ejected out to the external surface.

It is difficult to indicate a type of nucleus responsible for $\beta$-decay in stellar atmospheres. The decay of a neutron, during which protons and anti-neutrinos are discharged along with electrons, cannot explain the very quick generation of energy from flaring stars; the period of half-decay of neutrons is of the order of $12 \mathrm{~min}$, i.e. comparatively great. In some respects $\beta$-decay of $\mathrm{He}^{6}$ nuclei is of interest. First, the period of the decay of $\mathrm{He}^{6}$ is not large - less than $1 \mathrm{sec}$. Secondly, the maximum of the energy of the decay electron is at $\mu \sim 3 \cdot 5$, i.e. $\mu^{2} \approx 10$. Third, the product of the decay of $\mathrm{He}^{6}$ is lithium, the anomalous content of which is established in many non-stationary stars. For example, in the atmospheres of $T$ Tauri stars lithium is 100 times more abundant than in the Sun (Bonsak and Greenstein, 1960, Wallerstein et al., 1965). We can parenthetically note that lithium cannot be discovered in the atmospheres of the nuclei of planetary nuclei. Because of the high temperature, lithium will be ionized there, and all of the lines connected with the basic energy level of Li II lie in the region $180 \AA$. Finally, helium is the second most abundant element in stars. That fact, however, does not mean that we admit the possibility of the short-lived isotope of helium $\mathrm{He}^{6}$ issuing directly from the interior of the stars. The nuclei of $\mathrm{He}^{6}$ are in all probability formed in the atmosphere of stars after the issue of interstellar substances.

Now back to the non-thermal radio emission of planetary nebulae. We do not yet possess strong proofs in favor of the synchrotron origin of this radiation; but it is possible to affirm that it must be synchrotron radiation. The analysis of the given observations indicates that the parameter $(\gamma)$ of the energy spectrum of relativistic electrons is unexpectedly large, of the order of 4-5, and even larger. This must be contrasted with that of the cosmic radiation of non-thermal origin where it is not greater than three. This means that the spectrum of the relativistic electrons, responsible for radiation in planetary nebulae, is nearly mono-energetic, or rather similar to a gaussian, i.e. close to the energy spectrum of the electrons of $\beta$-decay. The generation of synchrotron radiation under the conditions of planetary nebulae $\left(H \simeq 10^{-3}-10^{-4}\right.$ gauss) requires that the energy of electrons should be $10^{8}-10^{9} \mathrm{eV}$, which is $2-3$ orders of magnitude more energy than electrons from $\beta$-decay. Such electrons may appear in a nebula either as a result of $\beta$-decay of nuclei unknown to us or by means of acceleration of electrons coming from $\beta$-decay already within the bounds of a nebula until the magnitude of $10^{8}-10^{9} \mathrm{eV}$ is reached. In the latter case the nucleus of a planetary nebula plays the part of injector of earlier accelerated particles breathing into the nebula electrons with energies of the order of $10^{6}-10^{7} \mathrm{eV}$, and the nebula plays the part of an accelerator, where the electrons are driven until the energy of $10^{8}-10^{9} \mathrm{eV}$ is reached. How and in what regions of the nebula this acceleration takes place is not clear to us, particularly if we take into consideration the fact that according to ideas developed by Gurzadian (1962) magnetic fields in the basic envelopes of planetary nebulae must be of the bipolar type. 
The difficulties connected with the means by which fast and relativistic electrons appear in planetary nebulae are great. Under such circumstances the possibility of highly energetic electrons generated in nebulae by other means should not be excluded. However this may occur, it is quite evident that the source of electrons must be the nuclei of nebulae. In that sense the classification of nuclei as objects with maximum non-stationarity is valid. In the present stage of the investigation of this matter we must, apparently, be satisfied with that conclusion according to which the appearance of fast electrons on the external regions of nuclei of nebulae and then their acceleration within the nebulae may explain the emission-line luminescence of planetary nebulae of comparatively low-temperature nuclei, as well as the fact of radio radiation having a synchrotron origin. The $\beta$-decay of some non-stable nuclei may be one of the possible mechanisms of the appearance of such electrons in the external regions of nuclei of nebulae.

\section{References}

Abell, G. (1966) Astrophys. J., 144, 259.

Aller, L.H. (1943) Astrophys. J., 97, 135.

Ambartsumian, V., Mustel, E., Severny, A., Sobolev, V. (1952) Theoretical Astrophysics, Moscow.

Ambartsumian, V. (1954) Comm. Burakan Obs., 13.

Bonsak, W. K., Greenstein, J.L. (1960) Astrophys. J., 131, 83.

Gurzadian, G.A. (1962) Planetary Nebulae, Moscow.

Gurzadian, G.A. (1965) Astrophys., 1, 319. (Also in Doklady Acad. Sci. U.S.S.R., 166, 1966, 53).

Gurzadian, G. A. (1966) Astrophys., 2, 217.

Kazarian, M. (1967) Comm. Burakan Obs., 38, 25.

Wallerstein, G., Herbig, G., Conti, P. (1965) Astrophys. J., 141, 610.

\section{DISCUSSION}

Reeves: Why do you want to get your high-energy electrons from $\beta$-decay? I cannot think of any way of making $\mathrm{He}^{6}$ in reasonable amounts in stellar atmosphere or nebulae. Electromagnetic acceleration of electrons would seem much more reasonable.

Gurzadian: It is a very complex question and I cannot answer now. $\mathrm{He}^{6}$ may be one of the possible, but not only source for the existence of the fast electrons in the outer regions of the star.

Salpeter: What was the assumed ratio of the rate of feeding energy into the $\beta$-decay electrons to the optical luminosity?

Gurzadian: Nearly 1000 if the optical thickness of the layer of fast electrons on the processes of Thomson scattering is $10^{-3}$.

Thompson: The subject of non-thermal radio emission from planetary nebulae has been mentioned several times both today and in previous discussions. In case any misunderstanding remains, I should like to emphasize that there is really no significant evidence that non-thermal emission occurs. All cases of deviation from a thermal spectrum which have been examined in detail have been found to result from source confusion, and there is no reason to suppose that any of the remaining cases will be different. 